\title{
EXPRESSION OF PRO- AND ANTIANGIOGENIC VEGF-A ISOFORMS AND SPLICING REGULATORY FACTORS IN BREAST CANCER
}

Rodrigo Castro1', Beatriz P. Bertelli1', Patrícia M. Biselli-Chicote¹, Dalísio S. Neto', José Luis E. Francisco1, Érika C. Pavarino', Eny M. Goloni-Bertollo'

'Unidade de Pesquisa em Genética e Biologia Molecular, Faculdade de Medicina de São José do Rio Preto, Hospital de Base - São José do Rio Preto (SP), Brazil.

Financial support: FAPESP (2010/19217-1); CNPQ, CAPES.

Objectives: The aim of this work was to analyze the expression of pro- and antiangiogenic Vascular Endothelial Growth Factor (VEGF-A) gene isoforms generated by alternative splicing in samples of breast cancer and non-tumor adjacent tissues, and to investigate the involvement of genes encoding the regulatory proteins SRPK1, SFRS1, SRSF5, and SRSF6 in the $V E G F-A$ gene alternative splicing. Methods: The expression of $V E G F-A_{165}, V E G F-A_{165} b$ isoforms and genes encoding the splicing regulatory proteins of 50 breast cancer samples and 43 adjacent non-tumor tissues were analyzed by real-time quantitative PCR. The values of relative quantification (RQ) in tumors were analyzed by Wilcoxon Signed Rank Test. Spearman correlation was used to evaluate the correlation between the expression of the genes encoding regulatory proteins and the VEGF- $A$ isoforms. Binary Logistic regression was used to analyze the association between the expression of $V E G F-A$ isoforms and metastasis. $\mathrm{P} \leq 0.05$ were considered significant. Results: The overexpression of $V E G F-A_{165}$ (median $R Q=7.7$, $\mathrm{p}<0.0001)$ and $V E G F-A_{165} b(\mathrm{RQ}=2.9, \mathrm{p}<0.0001)$ isoforms was observed in breast tumors compared to adjacent non-tumor tissues. The expression of SRPK1, SFRS1, SRSF5, and SRSF6 genes was significantly increased in breast tumors compared to non-tumor tissues ( $\mathrm{p}<0.0001)$. The expression of SFRS1, SRSF6, SRSF5 and SRPK1 were positively correlated with both isoforms of $V E G F-A$. Down-expression of antiangiogenic isoform $V E G F-A_{165} b$ was significantly associated with metastasis $(\mathrm{OD}=4.93 ; 95 \% \mathrm{CI} 1.03-23.63 ; \mathrm{p}=0.03)$. Conclusion: The overexpression of both pro- and antiangiogenic VEGF- $A$ isoforms in breast cancer can influence in the treatment of this tumor type, because the currently used anti-VEGF-A therapies target both isoforms, which could prevent the antiangiogenic activity of VEGF-A $\mathrm{A}_{165}$. The splicing regulatory factors SRSF1, SRSF6, SRSF5, and SRPK1 can contribute to alternative splicing of the VEGF-A gene. The expression of antiangiogenic isoform $V E G F-A_{165} b$ is a relevant factor for the prognosis of patients with breast cancer. 